\title{
Analysis of Adiponectin Gene Polymorphisms in Chinese Population with Systemic Lupus Erythematosus
}

\author{
Wen Liang Fang, ${ }^{1}$ Bin Zhou, ${ }^{2}$ Yan Yun Wang, ${ }^{1}$ Yu Chen,, ${ }^{2}$ and Lin Zhang, 2 \\ ${ }^{1}$ Department of Immunology, West China School of Preclinical and Forensic Medicine, Sichuan University, Chengdu 610041, China \\ ${ }^{2}$ Laboratory of Molecular Translational Medicine, West China Second University Hospital, Sichuan University, Chengdu 610041, China
}

Correspondence should be addressed to Lin Zhang, zhanglin@scu.edu.cn

Received 11 January 2010; Accepted 15 March 2010

Academic Editor: George C. Tsokos

Copyright ( $\odot 2010$ Wen Liang Fang et al. This is an open access article distributed under the Creative Commons Attribution License, which permits unrestricted use, distribution, and reproduction in any medium, provided the original work is properly cited.

\begin{abstract}
Systemic lupus erythematosus (SLE) is a prototypic systemic autoimmune disease. Adiponectin is an adipocyte-derived cytokine with anti-inflammatory, antidiabetic, and antiatherogenic properties. No study has reported on the association between adiponectin (ADIPOQ) gene and SLE. Our aim is to investigate the association between single-nucleotide polymorphisms in ADIPOQ gene and SLE. We examined 179 SLE patients and 237 age- and gender-matched controls from Sichuan province in China. Genotypes were determined using polymerase chain reaction-restriction fragment length polymorphism and DNA sequencing. Results show that there was no significant difference in the allele frequencies of $\mathrm{rs} 1501299(P=.311, \mathrm{OR}=1.17,95 \%$ CI: 0.86-1.59) and $\operatorname{rs} 2241766(P=.929, \mathrm{OR}=0.99,95 \% \mathrm{CI}: 0.74-1.33)$ in ADIPOQ gene between SLE patients and controls. The same results were seen in their genotypes $(P<.05)$. The allele frequencies of rs 1501299 and rs 2241766 polymorphisms of ADIPOQ may not be associated with SLE risk.
\end{abstract}

\section{Introduction}

Systemic lupus erythematosus (SLE) is a multisystem, complex autoimmune disease characterized by autoantibody production and tissue injury. The formation of immune complexes with these autoantibodies and their deposition in multiple organs contribute to eventual end-organ damage. The disease commonly affects young women and occurs with different frequencies in racial and ethnic groups [ 1 , 2]. Although many studies have shown that environmental and genetic factors contribute to SLE, its etiopathogenisis remains unclear $[3,4]$.

Adiponectin, a product of the adiponectin (ADIPOQ) gene, is the most abundant human adipose-specific protein [5]. It belongs to a family of adipocytokines, including adiponectin, leptin, resistin, and visfatin [6]. Adipocytokines are soluble mediators derived mainly from adipocytes and are thought to play an important role in inflammation and immunity [7]. Different adiponectin levels were found to be related to risks of type 2 diabetes, atherosclerosis, and coronary artery disease, among others $[8,9]$. Similarly, plasma, urine, and renal levels and expression of adiponectin were found to be associated with insulin resistance, body mass index, c-reactive protein, and nephritis in patients with SLE $[10,11]$. Although adiponectin has been suggested as a biomarker for SLE and SLE nephritis, other potential factors may prompt its elevation in SLE [12]. Thus, adiponectin has not been validated as a true SLE biomarker.

A group of researchers recently found that plasma adiponectin levels are significantly lower in obesity, type 2 diabetes, hypertension, atherosclerosis, and other diseases [13], while in SLE, diabetic nephropathy, and chronic renal failure, the plasma and urine levels of adiponectin are significantly raised $[11,14]$. These phenomena exist for a number of reasons. First, insulin resistance in obesity, type 2 diabetes, hypertension, atherosclerosis, and other diseases is a process of attaining a state of energy equilibrium. Low serum adiponectin levels can be viewed as a pathophysiological process in the initial event of insulin resistance. The negative energy balance of insulin resistance in SLE can stimulate the secretion of adiponectin to overcome the inhibiting factor, and serum adiponectin levels increase 
significantly. Second, proteinuria in SLE patients causes simultaneous loss of large amounts of plasma albumin, certain immunoglobulin, complement components, and metal-binding proteins. This loss may cause degradation of the adiponectin enzymes and clear adiponectin disorder. Third, as a protective cytokine, adiponectin may reduce the metabolic disorder and endothelial vascular injury caused by other risk factors in SLE patients. It stimulates secretion of adiponectin to overcome the inhibiting factor, so that serum adiponectin level is significantly raised.

Adiponectin levels have a strong genetic component, with an additive genetic heritability of $46 \%$ [15]. The ADIPOQ gene consists of three exons and two introns spanning a $17-\mathrm{kb}$ region and has been located on chromosome 3q27 [16]. The ADIPOQ gene was found to be the only major gene responsible for plasma adiponectin [17]. While majority of studies on ADIPOQ gene polymorphisms have focused on coronary artery disease, type 2 diabetes, and obesity [18-21], no study, to date, has examined the association between single-nucleotide polymorphisms (SNPs) of the ADIPOQ gene and SLE. Therefore, in this study, we investigated two SNPs (rs1501299 and rs2241766) in ADIPOQ gene and their possible association with SLE susceptibility in the Chinese Han population.

\section{Materials and Methods}

2.1. Subjects. A total of 179 unrelated Han SLE patients from West China Hospital, Sichuan University, were enlisted from July 2006 to March 2009. Patients (18 males and 161 females) with an average age of $33.5 \pm 13.1$ years had typical clinical SLE symptoms and were diagnosed according to the American Rheumatism Association Criteria for SLE classification [22]. A total of 237 controls (24 men and 213 women) with an average age of $34.5 \pm 11.8$ years had healthy blood with no family history of SLE. The controls were taken from the same geographic areas as the patients, whose gender ratio and mean ages were matched with the SLE group. A written informed consent was obtained from all the subjects, and the study was performed with the approval of the ethics committee of the Chinese Human Genome.

2.2. Genotyping of ADIPOQ. Genomic DNA was extracted from peripheral blood cells using an extraction kit (Bioteke Corporation, Perking, China) according to the manufacturer's instructions. Polymorphisms of adiponectin gene were identified using polymerase chain reaction-restriction fragment length polymorphism (PCR-RFLP) analysis. The primer sequences and reaction conditions are shown in Table 1. To further confirm the genotyping results, PCRamplified DNA samples were examined by DNA sequencing.

2.3. Statistical Analysis. Genotype and allele frequencies of rs1501299 and rs2241766 in ADIPOQ gene were compared in two groups using a $\chi^{2}$ test and Fisher's exact test. Odds ratio $(\mathrm{OR})$ and $95 \%$ confidence intervals $(\mathrm{CI})$ were calculated to assess the relative risk conferred by a particular allele and genotype. Demographic and clinical data between groups were analyzed by $\chi^{2}$ test and the Student's $t$-test. HardyWeinberg equilibrium was tested with a goodness of fit $\chi^{2}$ test with one degree of freedom to compare the observed genotype frequencies among subjects with the expected genotype frequencies. Statistical significance was assumed at the $P<.05$ level. The Statistical Package for Social Sciences (SPSS 11.5 Chicago, IL, U.S.A.) was used for all of the statistical analyses.

\section{Results}

The genotype and allele frequencies of rs1501299 and rs2241766 polymorphisms are shown in Table 2 . The genotyping results by PCR-RFLP and DNA sequencing were $100 \%$ concordant. The frequencies of the AA, AC, and CC genotypes of rs 1501299 were $5.6 \%, 47.5 \%$, and $46.9 \%$ in patients and $6.8 \%, 38.8 \%$, and $54.4 \%$ in the controls, respectively. The frequencies of the $\mathrm{A}$ and $\mathrm{C}$ alleles of rs 1501299 were $29.3 \%$ and $70.7 \%$ in patients and $26.2 \%$ and $73.8 \%$ in the controls, respectively. The frequencies of the GG, GT, and TT genotypes of rs 2241766 were $8.4 \%, 46.4 \%$, and $45.3 \%$ in patients and $8.0 \%, 47.7 \%$, and $44.3 \%$ in the controls, respectively. The frequencies of $\mathrm{G}$ and $\mathrm{T}$ alleles of rs 2241766 were $31.6 \%$ and $68.4 \%$ in patients and $31.9 \%$ and $68.1 \%$ in the controls, respectively. No significant difference was observed in the allele frequencies of the rs1501299 and rs 2241766 polymorphisms between the patients and controls (for rs1501299: $P=.311$, OR $=1.17,95 \%$ CI: $0.86-1.59$; for rs2241766: $P=.929, \mathrm{OR}=0.99,95 \%$ CI: 0.74-1.33). The same results were seen in the genotypes (Table 2).

\section{Discussion}

To the best of our knowledge, this is the first study that investigated the association between the rs2241766 and rs 1501299 polymorphisms of ADIPOQ gene and SLE. In this study, no significant difference was found in the distribution of ADIPOQ gene polymorphisms between SLE patients and controls, suggesting that ADIPOQ gene polymorphisms may not be a significant contributor to SLE susceptibility.

Adiponectin is an adipocyte-derived peptide expressed exclusively in adipocytes. Adiponectines are also called gelatin-binding protein-28 (GBP28), AdipoQ, ACRP30, or apM1. Low plasma adiponectin concentration is associated with metabolic disorders and an increased risk of cardiovascular events [23]. However, circulating adiponectin levels have been reported to be significantly increased in patients with SLE, while patients with high plasma adiponectin had poor prognosis in lupus nephritis $[10,24]$. Serum levels of adiponectin were significantly and inversely correlated with insulin resistance in SLE patients. Elevated levels of adiponectin in SLE suggest the possible involvement of adiponectin in insulin resistance and alteration of insulin sensitivity [25].

Some studies showed that the plasma level, expression, and biological effects of adiponectin are associated with polymorphism in the ADIPOQ gene [26-28]. Polymorphisms in the ADIPOQ gene have also been shown to correlate 
TABLE 1: Primer sequences and reaction conditions for genotyping adiponectin polymorphisms.

\begin{tabular}{|c|c|c|c|c|c|c|}
\hline Gene & Location & Primer sequence & Product (bp) & $\begin{array}{l}\text { Annealing } \\
\text { temperature }\left({ }^{\circ} \mathrm{C}\right)\end{array}$ & $\begin{array}{l}\text { Restriction } \\
\text { enzyme }\end{array}$ & Allele (bp) \\
\hline \multirow{2}{*}{ rs1501299 } & \multirow{2}{*}{ Intron-2 } & 5'-GTCTAGGCCTTAGTTAATAATGAAGG-3' & \multirow{2}{*}{106} & \multirow{2}{*}{56} & \multirow{2}{*}{ STU I } & A (106) \\
\hline & & 5'-GTGAGAAAGGAGATCCAGGTAA-3' & & & & $\mathrm{C}(80,26)$ \\
\hline \multirow{2}{*}{ rs2241766 } & \multirow{2}{*}{ Exon-2 } & 5'-TGGACGGAGTCCTTTGTAGG-3' & \multirow{2}{*}{161} & \multirow{2}{*}{56} & \multirow{2}{*}{ Sma I } & $\mathrm{G}(134,27)$ \\
\hline & & 5'-TTGAGTCGTGGTTTCCTGGT-3' & & & & $\mathrm{T}(161)$ \\
\hline
\end{tabular}

TABLE 2: Genotype and allele frequencies of rs2241766 and rs1501299 in ADIPOQ between patients with asthma and controls.

\begin{tabular}{|c|c|c|c|c|}
\hline Polymorphisms & $\begin{array}{c}\text { Patients } \\
n=179(\%)\end{array}$ & $\begin{array}{c}\text { Controls } \\
n=237(\%)\end{array}$ & OR (95\% CI) & $P$ \\
\hline \multicolumn{5}{|l|}{$\begin{array}{l}\text { rs1501299 } \\
\text { genotypes }\end{array}$} \\
\hline $\mathrm{AA}$ & $10(5.6)$ & $16(6.8)$ & 1.00 (Ref) & \\
\hline $\mathrm{AC}$ & $85(47.5)$ & $92(38.8)$ & $0.68(0.29-1.57)$ & .362 \\
\hline $\mathrm{CC}$ & $84(46.9)$ & $129(54.4)$ & $0.96(0.42-2.22)$ & .923 \\
\hline \multicolumn{5}{|l|}{ alleles } \\
\hline A & $105(29.3)$ & $124(26.2)$ & 1.00 (Ref) & \\
\hline $\mathrm{C}$ & $253(70.7)$ & $350(73.8)$ & $1.17(0.86-1.59)$ & .311 \\
\hline $\begin{array}{r}\text { rs2241766 } \\
\text { genotypes }\end{array}$ & \multicolumn{3}{|c|}{ rs2241766 } & \\
\hline GG & $15(8.4)$ & $19(8.0)$ & 1.00 (Ref) & \\
\hline GT & $83(46.4)$ & $113(47.7)$ & $1.08(0.52-2.24)$ & .847 \\
\hline TT & $81(45.3)$ & $105(44.3)$ & $1.023(0.49-2.14)$ & .951 \\
\hline \multicolumn{5}{|l|}{ alleles } \\
\hline G & $113(31.6)$ & $151(31.9)$ & 1.00 (Ref) & \\
\hline $\mathrm{T}$ & $245(68.4)$ & $323(68.1)$ & $0.99(0.74-1.33)$ & .929 \\
\hline
\end{tabular}

with adiponectin serum levels, in which the expression of the G allele at SNP rs2241766 was consistently higher than the $\mathrm{T}$ allele among all study subjects [28]. Moreover, Hara et al. reported that the C allele at SNP rs1501299 is inversely associated with lower plasma adiponectin concentration in Japanese population. Yang and Chuang reported that the A allele at SNP rs1501299 is associated with lower serum adiponectin concentration in Italians $[23,27]$. There are other factors that have been shown to regulate adiponectin levels. A Mediterranean diet or a diet rich in whole grain and fat was shown to produce increased adiponectin levels $[29,30]$. Physical activity was also shown to influence adiponectin, and high levels of physical activity could elevate adiponectin levels [31].

Based on these studies, we started this study with the hypothesis that the ADIPOQ gene polymorphisms may be one of the genetic factors that affect SLE susceptibility. However, our results show that the SNP rs2241766 and rs1501299 in the ADIPOQ gene had no association with SLE in the Chinese Han population. No significant difference was found in allele or genotype frequencies between SLE patients and the controls in terms of the selected SNPs. This finding is contrary with our hypothesis and suggests that this polymorphism should be tested in groups of different ethnic origins.

Although deciphering the reasons for failure and finding a positive association is difficult, several possibilities should necessarily be considered. First, SLE is a multifactorial disease; different individuals could be exposed to various environmental factors and genetic susceptibility might lead to different results. Second, the disparity between our results and assumptions may be due to the relatively small number of SLE patients tested. A variation in ADIPOQ gene may contribute to susceptibility to SLE, but the effect should be minimal. Large, varied populations of SLE patients should be tested to avoid a statistical false negative. Third, although the investigated SNPs (rs2241766 and rs1501299) do not affect susceptibility to SLE in our study, further research on more SNPs in the ADIPOQ gene is needed to exclude the role of the adiponectin gene polymorphisms as a possible susceptibility factor for SLE. Finally, the inadequate study design, such as nonrandom sampling, should also be considered. The possibility of selection bias from the hospital-based case-control study is a relevant issue. Nevertheless, the results of this study provide additional information and motivation for further research 
into the association of ADIPOQ gene polymorphisms and SLE.

In conclusion, we found that ADIPOQ gene polymorphisms were not associated with the risk of SLE in the Chinese Hans population. Further studies are needed to explore the complicated interaction between environmental factors and ADIPOQ gene polymorphisms in terms of susceptibility to SLE, especially in ethnically diverse populations.

\section{Acknowledgments}

This work was funded by grants from the China Medical Board (88-486). The authors are most grateful to all subjects who had willingly participated and encouraged them to proceed with this study. None of the authors has any potential financial conflict of interest related to this manuscript.

\section{References}

[1] A. Rahman and D. A. Isenberg, "Systemic lupus erythematosus," The New England Journal of Medicine, vol. 358, no. 9, pp. 929-939, 2008.

[2] J. Wang, S. Yang, J. J. Chen, et al., "Systemic lupus erythematosus: a genetic epidemiology study of 695 patients from China," Archives of Dermatological Research, vol. 298, no. 10, pp. 485491, 2007.

[3] S. P. Ardoin and D. S. Pisetsky, "Developments in the scientific understanding of lupus," Arthritis Research and Therapy, vol. 10 , no. 5 , p. $218,2008$.

[4] M. Molokhia and P. Mckeigue, "Systemic lupus erythematosus: genes versus environment in high risk populations," Lupus, vol. 15, no. 11, pp. 827-832, 2006.

[5] K. Maeda, K. Okubo, I. Shimomura, T. Funahashi, Y. Matsuzawa, and K. Matsubara, "cDNA cloning and expression of a novel adipose specific collagen-like factor, apM1 (AdiPose Most abundant Gene transcript 1)," Biochemical and Biophysical Research Communications, vol. 221, no. 2, pp. 286-289, 1996.

[6] H. Tilg and A. R. Moschen, "Adipocytokines: mediators linking adipose tissue, inflammation and immunity," Nature Reviews Immunology, vol. 6, no. 10, pp. 772-783, 2006.

[7] N. Ouchi, S. Kihara, Y. Arita, et al., "Novel modulator for endothelial adhesion molecules: adipocyte-derived plasma protein adiponectin," Circulation, vol. 100, no. 25, pp. 24732476, 1999.

[8] J. Kawano and R. Arora, "The role of adiponectin in obesity, diabetes, and cardiovascular disease," Journal of the CardioMetabolic Syndrome, vol. 4, no. 1, pp. 44-49, 2009.

[9] S. Li, H. J. Shin, E. L. Ding, and R. M. Van Dam, "Adiponectin levels and risk of type 2 diabetes: a systematic review and metaanalysis," Journal of the American Medical Association, vol. 302, no. 2, pp. 179-188, 2009.

[10] C. P. Chung, A. G. Long, J. F. Solus, et al., "Adipocytokines in systemic lupus erythematosus: relationship to inflammation, insulin resistance and coronary atherosclerosis," Lupus, vol. 18, no. 9, pp. 799-806, 2009.

[11] B. H. Rovin, H. Song, L. A. Hebert, et al., "Plasma, urine, and renal expression of adiponectin in human systemic lupus erythematosus," Kidney International, vol. 68, no. 4, pp. 18251833, 2005.
[12] B. H. Rovin, D. J. Birmingham, H. N. Nagaraja, C. Y. Yu, and L. A. Hebert, "Biomarker discovery in human SLE nephritis," Bulletin of the NYU Hospital for Joint Diseases, vol. 65, no. 3, pp. 187-193, 2007.

[13] M. Adamczak, A. Wiecek, T. Funahashi, J. Chudek, F. Kokot, and Y. Matsuzawa, "Decreased plasma adiponectin concentration in patients with essential hypertension," American Journal of Hypertension, vol. 16, no. 1, pp. 72-75, 2003.

[14] C. Zoccali, F. Mallamaci, G. Tripepi, et al., "Adiponectin, metabolic risk factors, and cardiovascular events among patients with end-stage renal disease," Journal of the American Society of Nephrology, vol. 13, no. 1, pp. 134-141, 2002.

[15] A. G. Comuzzie, T. Funahashi, G. Sonnenberg, et al., "The genetic basis of plasma variation in adiponectin, a global endophenotype for obesity and the metabolic syndrome," Journal of Clinical Endocrinology and Metabolism, vol. 86, no. 9, pp. 4321-4325, 2001.

[16] M. Takahashi, Y. Arita, K. Yamagata, et al., "Genomic structure and mutations in adipose-specific gene, adiponectin," International Journal of Obesity, vol. 24, no. 7, pp. 861-868, 2000.

[17] I. M. Heid, P. Henneman, A. Hicks, et al., "Clear detection of ADIPOQ locus as the major gene for plasma adiponectin: results of genome-wide association analyses including 4659 European individuals," Atherosclerosis, vol. 208, no. 2, pp. 412420, 2010.

[18] G. Mohammadzadeh and N. Zarghami, "Associations between single-nucleotide polymorphisms of the adiponectin gene, serum adiponectin levels and increased risk of type 2 diabetes mellitus in Iranian obese individuals," Scandinavian Journal of Clinical and Laboratory Investigation, vol. 69, no. 7, pp. 764771, 2009.

[19] L. Li, R. Wu, and J. Zhao, "Adiponectine gene +45T/G and $+276 \mathrm{G} / \mathrm{T}$ polymorphism and antipsychotic-induced weight gain," Zhong Nan Da Xue Xue Bao Yi Xue Ban, vol. 34, no. 8, pp. 693-696, 2009.

[20] K. Tsuzaki, K. Kotani, N. Nagai, et al., "Adiponectin gene single-nucleotide polymorphisms and treatment response to obesity," Journal of Endocrinological Investigation, vol. 32, no. 5, pp. 395-400, 2009.

[21] L. Foucan, N. Ezourhi, S. Maimaitiming, et al., "Adiponectin multimers and ADIPOQ T45G in coronary artery disease in Caribbean type 2 diabetic subjects of African descent," Obesity. In press.

[22] E. M. Tan, A. S. Cohen, J. F. Fries, et al., "The 1982 revised criteria for the classification of systemic lupus erythrematosus," Arthritis and Rheumatism, vol. 25, no. 11, pp. 1271-1277, 1982.

[23] W.-S. Yang and L.-M. Chuang, "Human genetics of adiponectin in the metabolic syndrome," Journal of Molecular Medicine, vol. 84, no. 2, pp. 112-121, 2006.

[24] J. B. De Sanctis, M. Zabaleta, N. E. Bianco, J. V. Garmendia, and L. Rivas, "Serum adipokine levels in patients with systemic lupus erythematosus," Autoimmunity, vol. 42, no. 4, pp. 272274, 2009.

[25] K.-E. Sada, Y. Yamasaki, M. Maruyama, et al., "Altered levels of adipocytokines in association with insulin resistance in patients with systemic lupus erythematosus," Journal of Rheumatology, vol. 33, no. 8, pp. 1545-1552, 2006.

[26] W.-S. Yang, P.-L. Tsou, W.-J. Lee, et al., "Allele-specific differential expression of a common adiponectin gene polymorphism related to obesity," Journal of Molecular Medicine, vol. 81, no. 7, pp. 428-434, 2003. 
[27] K. Kara, P. Boutin, Y. Mori, et al., "Genetic variation in the gene encoding adiponectin is associated with an increased risk of type 2 diabetes in the Japanese population," Diabetes, vol. 51, no. 2, pp. 536-540, 2002.

[28] T. I. Pollin, K. Tanner, J. R. O'Connell, et al., "Linkage of plasma adiponectin levels to $3 \mathrm{q} 27$ explained by association with variation in the APM1 gene," Diabetes, vol. 54, no. 1, pp. 268-274, 2005.

[29] C. S. Mantzoros, C. J. Williams, J. E. Manson, J. B. Meigs, and F. B. $\mathrm{Hu}$, "Adherence to the Mediterranean dietary pattern is positively associated with plasma adiponectin concentrations in diabetic women," American Journal of Clinical Nutrition, vol. 84, no. 2, pp. 328-335, 2006.

[30] C. S. Mantzoros, T. Li, J. E. Manson, J. B. Meigs, and F. B. $\mathrm{Hu}$, "Circulating adiponectin levels are associated with better glycemic control, more favorable lipid profile, and reduced inflammation in women with type 2 diabetes," Journal of Clinical Endocrinology and Metabolism, vol. 90, no. 8, pp. 4542-4548, 2005.

[31] Z. Yu, X. Ye, J. Wang, et al., "Associations of physical activity with inflammatory factors, adipocytokines, and metabolic syndrome in middle-aged and older chinese people," Circulation, vol. 119, no. 23, pp. 2969-2977, 2009. 


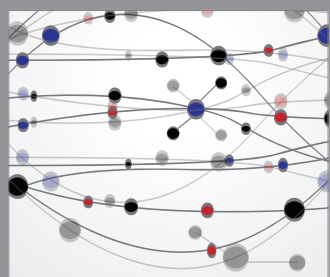

The Scientific World Journal
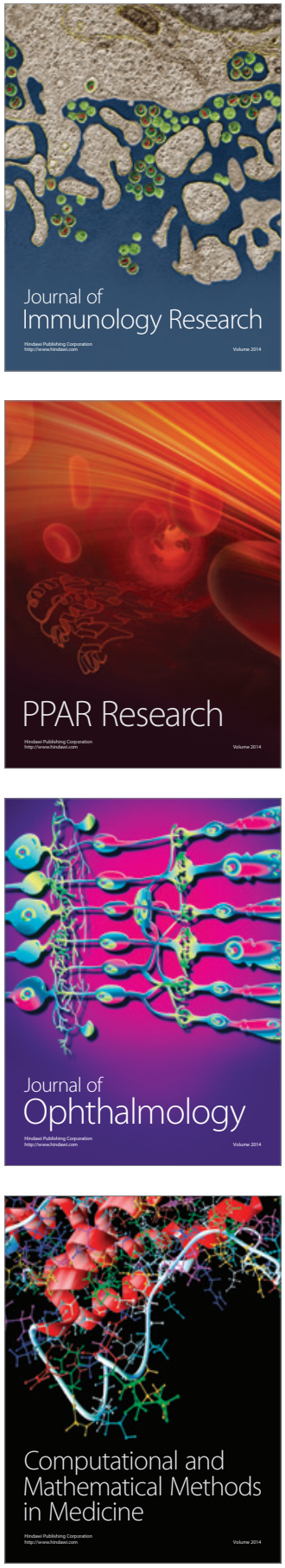

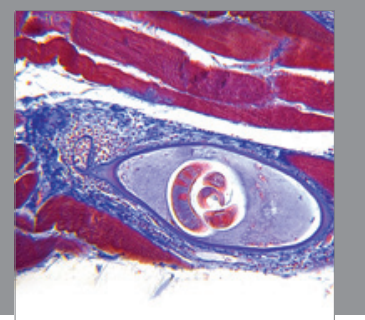

Gastroenterology

Research and Practice
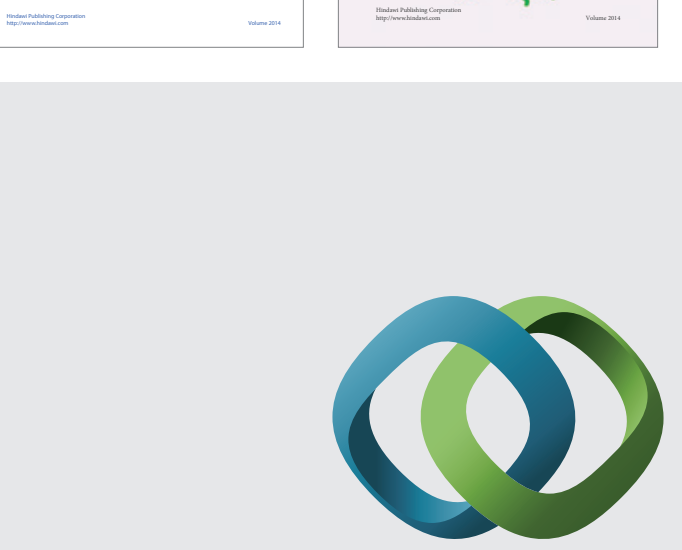

\section{Hindawi}

Submit your manuscripts at

http://www.hindawi.com
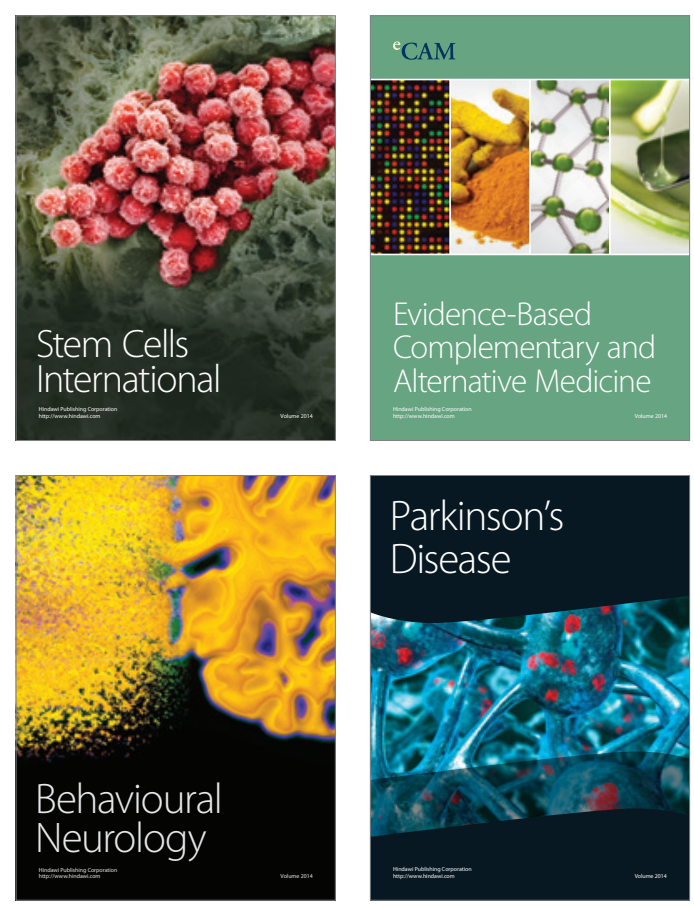

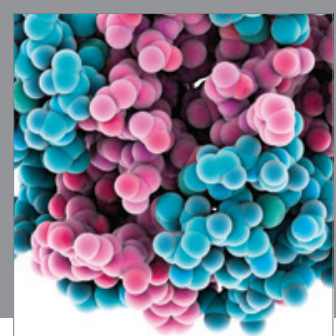

Journal of
Diabetes Research

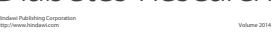

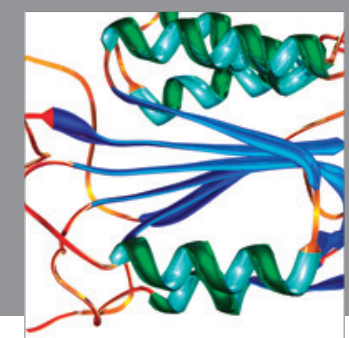

Disease Markers
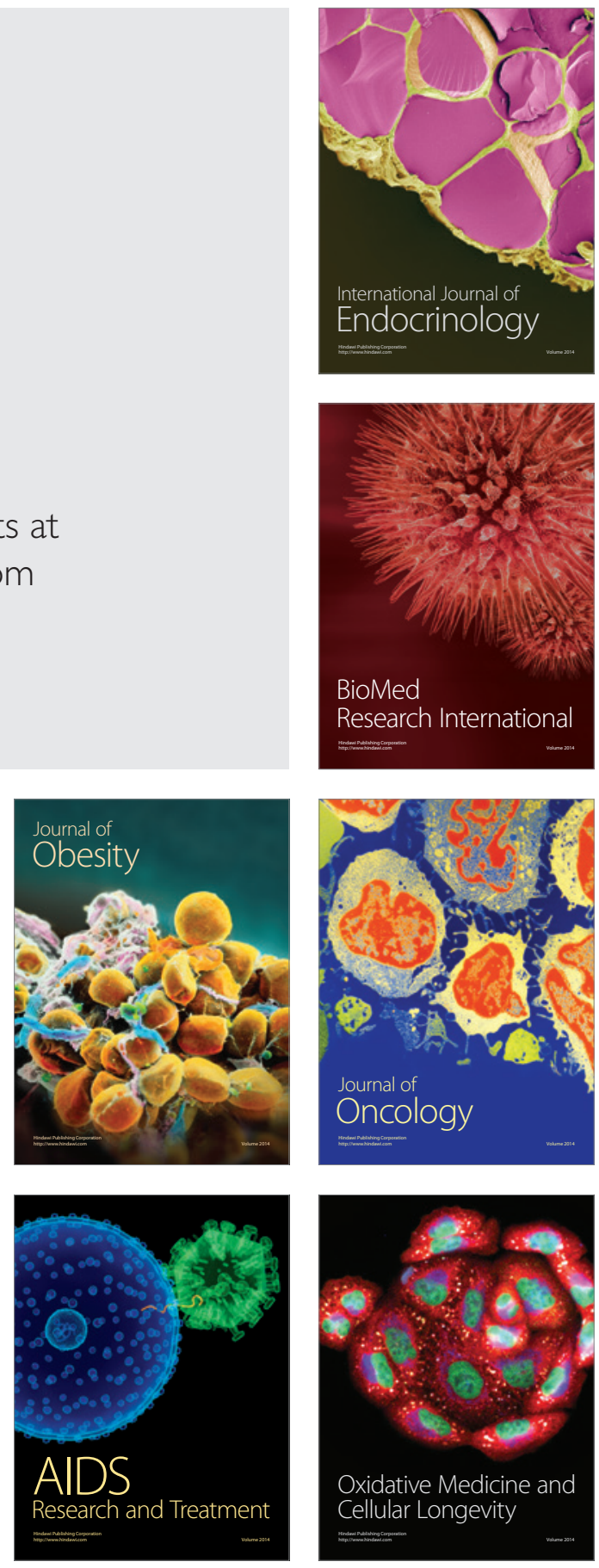